\title{
Ist der Regressterror jetzt wirklich vorbei?
}

— Ein „Ende des Regressterrors“ hat jüngst die Freie Ärzteschaft auf ihrer Jahresdelegiertenversammlung gefordert. Als Reaktion hat Bundesgesundheitsminister Daniel Bahr (FDP) mitgeteilt, dass Regresse angeblich kein Thema mehr sind. Eine solche Bedrohung soll demnach für den Rest dieser Legislaturperiode praktisch keine Rolle mehr spielen.

„In den nächsten zwei Jahren wird es zu fast keinen Arznei- und Heilmittel-Regressen mehr kommen", sagte der Minister bei einem Werkstattgespräch in der Kassenärztlichen Vereinigung (KV) Nordrhein zu. Laut Bahr könne das kommende Versorgungsstrukturgesetz so die Regressangst nehmen und unter anderem auch positiven Einfluss auf den drohenden Ärztemangel nehmen, weil für Ärzte in ländlichen Regionen keine Mengenbegrenzung mehr bei ärztlichen Leistungen vorgesehen sei, sondern künftig alle Leistungen voll bezahlt würden.

\section{MMW Kommentar}

Die sehr mutig eindeutigen Aussagen des Gesundheitsministers sind erfreulich, aber mit einigen Fragezeichen belastet. Wenn künftig tatsächlich ein Arznei- oder Heilmittelregress nicht mehr möglich ist, weil - wie im Versorgungsgesetz (GKV VStG) vorgesehen - bei einer Richtgrößenüberschreitung zunächst eine Beratung stattfindet und erst dann, wenn die Inhalte dieser Beratung nicht umgesetzt werden, ein Regress folgen kann, stellt sich zunächst die Frage, für welchen Zeitraum das gilt? Werden Ärztinnen und Ärzte schon so behandelt, die vor 2012 statistisch auffällig geworden sind? Was passiert, wenn eine Beratung stattfindet, wegen der starken zeitlichen Verzögerung bei der Erfassung der Verordnungen für Arznei- und Heilmitteldaten deren Inhalte von der Ärztin/dem Arzt aber erst zwei oder drei Quartale später befolgt werden können? Was passiert, wenn eine solche Beratung dazu führt, dass - trotz der Überschreitung der Richtgrößen - keine Unwirtschaftlichkeit bei der Verordnung erkennbar ist und in den Folgequartalen folgerichtig weiter RichtgröBenüberschreitungen vorliegen? Erhält der Betreffende dann einen "Persilschein"? Die Aussagen des Ministers lassen so gesehen eine nicht unerhebliche Unkenntnis über die tatsächliche Situation vor Ort erkennen. Das zeigt auch die Aussage zur Honorierung von "Landärzten“. Wenn es künftig dort keine Honorarbegrenzungen mehr geben soll, wer finanziert das? Die Kassen haben bereits ihre Zahlungsbereitschaft negiert! Und wer entscheidet, wo das Land anfängt und die Stadt aufhört?

Einfacher wäre es sicherlich, solche Zusagen ohne weiteren zusätzlichen bürokratischen Aufwand zu realisieren, $z$. B. indem man die Richtgrößenprüfungen abschafft und die hausärztliche Vergütung flächendeckend nach dem Prinzip der "alten" HZV-Verträge gesetzlich erzwingt.

\section{Kassen sparen überall, aber nicht bei sich selbst!}

- Der Bundesrechnungshof hat bei stichprobenartigen Überprüfungen von Mietverträgen gesetzlicher Krankenkassen festgestellt, dass viele dieser Verträge mit Mieten über dem ortsüblichen Niveau oder mit Laufzeiten von bis zu 20 Jahren ohne ordentliches Kündigungsrecht abgeschlossen wurden. Oft wurde auch eine viel zu große Fläche angemietet. Aus der daraus entstandenen Unterdeckung und aus Leerständen entstand so einer Kasse ein finanzieller Nachteil von mindestens 6 Mio. Euro bis Ende 2010.

Nach den Recherchen der Bundesbehörde fehlt oft auch der wirtschaftliche Nutzen von Kassenfusionen. Bei fast allen unter- suchten Zusammenschlüssen sind die Verwaltungsausgaben der Kassen im Jahr der Fusion um bis zu $18 \%$ gestiegen, nicht zuletzt, weil sich bei der Hälfte dieser Fusionen die Vorstandsgehälter um bis zu 25\% erhöht hätten. So soll eine Krankenkasse Vorständen, die nach der Fusion aus ihrem Amt ausschieden, Abfindungen in Millionenhöhe gewährt haben.

\section{MMW Kommentar}

Neben den Traumgehältern sind jetzt auch die Pensionen der Krankenkassenchefs bekannt geworden. Wie die "Bild"-Zeitung bereits 2005 berichtete, haben Dutzende von Krankenkassenchefs Pensionsansprüche zum Teil in Höhe von mehr als 7000 Euro. Viele Krankenkassen-, aber auch Ärztefunktionäre können mit Pensionen von bis zu $71,75 \%$ des Gehaltes rechnen. So hat der frühere Chef des AOK-Bundesverbandes einen Pensionsanspruch in Höhe der Hälfte seines letzten Jahresgehaltes und damit von rund 7100 Euro im Monat.

Den Vogel schießt allerdings die KBV ab. Sie zahlt für ihren Vorstandschef zusätzlich zu seinem Gehalt von aktuell 350000 Euro pro Jahr noch 54000 Euro in eine Altersvorsorge. Daraus resultieren rund 6000 Euro Monatsrente ab dem 65. Lebensjahr. 